Dhaka Univ. J. Biol. Sci. 28(2): 195-209, 2019 (July)

\title{
6MYCELIAL GROWTH VARIATION AND COMPATIBILITY OF THE SELECTED ISOLATES OF BIPOLARIS SOROKINIANA (SACC.) SHOEMAKER
}

\author{
Mst. Selina Momtaz ${ }^{1}$, Shamim Shamsi* and Tapan Kumar Dey ${ }^{2}$ \\ Department of Botany, University of Dhaka, Dhaka-1000, Bangladesh
}

Key words: Cultural variation, Mycelial compatibility, Bipolaris sorokiniana, Leaf blight, Wheat

\begin{abstract}
Bipolaris sorokiniana (Sacc.) Shoemaker is a phytopathogenic fungus, causal agent of leaf blight disease of wheat. This fungus is difficult to control because of its immense cultural, morphological, physiological, pathogenic and genetic variability. The aim of this investigation was to study the cultural variability of the selected isolates of $B$. sorokiniana by means of mycelial compatibility. One hundred fifty isolates of B. sorokiniana from eight districts (Dhaka, Gazipur, Dinajpur, Joypurhat, Pabna, Sirajgonj, Kushtia and Chuadanga) of Bangladesh were studied and cultural variation was characterized on the basis of colony color and texture on PDA medium. They were - Black-Mat (B-M), Black-Fluffy (B-F), Ash-Mat (A-M), Brownish Ash-Fluffy (Br. A-F), Blackish Ash-Mat (Bl.AM), Whitish Ash-Mat (W. A-M), Greenish Ash-Fluffy (G. A-F) and Pinkish White-Mat (P.W-M). To compare and understand cultural variability, mycelial compatibility test was done among the cultural groups. Results revealed that selected isolates of different cultural groups showed incompatible reaction. Incompatibility was also noticed between the isolates of same district and different districts.
\end{abstract}

\section{Introduction}

Wheat (Triticum aestivum L.) is the second most important cereal crop next to rice in Bangladesh. During 2016 - 2017, total wheat production was 1.311 million tons from 0.415 million hectares of land. The country needs 5.5 million tons wheat, which are imported every year ${ }^{(1)}$. Still the yield level is much lower than the potential yield. Diseases play an important role in lowering yield in the country. Wheat is attacked by at least 20 different diseases in Bangladesh, of which five are considered as major diseases, they are Bipolaris leaf blight - (Bipolaris sorokiniana), leaf rust (Puccinia recondita), seedling blight (Bipolaris sorokiniana), foot and root rot (Sclerotium rolfsii Tode) and black point [B. sorokiniana, Alternaria alternata (Fr) Keissler, Curvularia lunata (Wakker) Boedijn and species of Fusarium Link] ${ }^{(2,3)}$. Sadat and Choi ${ }^{(4)}$ reported wheat blast caused by Pyricularia graministritici and P. oryzae from Bangladesh.

\footnotetext{
*Author for correspondence: <prof.shamsi@gmail.com>. ${ }^{1} \mathrm{~A}$ part of Ph.D. Thesis of first author (MSM). Present address: Department of Botany, Jagannath University, Dhaka-1100, ${ }^{2}$ Senior Program Specialist (Crops) KGF, BARC Complex, Farmgate, Dhaka \& Former Director, BARI, Gazipur, Bangladesh.
} 
Bipolaris sorokiniana (Sacc.) Shoemaker, teleomorph, Cochliobolus sativus (Ito and Kurib.) Drechsler ex Dastur, is the most important biotic constraint affecting wheat production in Bangladesh ${ }^{(5)}$. This fungus acts as a causal agent for various diseases like head blight, seedling blight, foliar blight/spot blotch, common root rot and black point of wheat, barley, other small cereal grains and grasses ${ }^{(6,7)}$. On an average, a South-Asian country loses $20 \%$ of crop yield through leaf blight of wheat ${ }^{(8)}$. Yield loss was estimated to be $15 \%$ in Bangladesh, $18-22 \%$ in India ${ }^{(9,10)}$.

The pathogen exhibits enormous cultural, morphological, physiological, pathological and molecular variability. Since B. sorokiniana asexually or clonally propagated, the chromosomal rearrangements may play a role in generating variation within pathogens and that processes responsible may be active during somatic growth and parasexual recombination ${ }^{(11)}$. Tinline ${ }^{(12)}$ reported that variability of $B$. sorokiniana was due to anastomosis and nuclear migration. Mycelial compatibility is the ability of two strains of filamentous fungi to anastomose colony and form one continuous colony, is synonymous with vegetative compatibility. Hyphal fusion between two individuals forms vegetative heterokaryons that result in genetically different nuclei. A sharp distinction must be maintained between two isolates in heterokaryon compatibility unless it is known that two strains not only anastomose but also form a stable heterokaryon. As an easy test for self-recognition, vegetative compatibility has been extremely useful in intraspecific strains comparisons ${ }^{(13)}$. Compatible pairing formed one confluent colony. Incompatible pairing produced a visible reaction in the interaction zone, if individuals differ in allelic specificity at heterokaryon incompatibility loci, the hyphal fusion cell is quickly compartmentalized and killed ${ }^{(14)}$.

Most of the high yielding varieties which are being grown on commercial scale in Bangladesh are found to be more or less susceptible to leaf blight (B. sorokiniana) disease. The reasons for lack of substantial durable resistance in the varieties may be attributed to presence of variability in the population(15). In order to develop the disease resistant and high yielding cultivars, it is imperative to analyze and understand the variability in the pathogen. The host plant resistance depends on the effectiveness of resistance against all biotypes or races of pathogen present in the region. Thus, studies on variability of $B$. sorokiniana have a greater significance in breeding for resistance against leaf blight of wheat. So, the present investigation was undertaken to study the cultural variability of isolates of $B$. sorokiniana by means of vegetative compatibility.

\section{Materials and Methods}

A total of 293 Bipolaris sorokiniana isolates were obtained from eight districts (Dhaka, Gazipur, Dinajpur, Joypurhat, Pabna, Sirajgonj, Kushtia and Chuadanga) of Bangladesh. BpLB infected samples of 21 wheat varieties were collected at grain filling stage and 
placed in clean brown paper bag, labeled properly and preserved at $4^{\circ} \mathrm{C}$ in refrigerator for studies.

The fungi were isolated from the samples following "Tissue planting method" on PDA medium ${ }^{(16)}$. First the diseased leaf samples were washed under flowing tap water. Small bits $\left(2^{2} \mathrm{~mm}\right)$ of diseased portion showing typical symptoms along with healthy leaf tissue were cut into pieces with the help of sterile blade and surface of the pieces were sterilized by dipping in 10\% Chlorox solution for 2 minutes and again washed in sterilized water thrice. Finally, the inocula were placed inside the folds of a sterile blotting paper to remove excess surface water. Then they were transferred to medium under aseptic conditions using laminar air flow. The inocula were placed in Petri plates containing PDA medium, each Petri plate contained $15 \mathrm{ml}$ of PDA medium with an additional of 1 drop (ca. $0.03 \mathrm{ml}$ ) of lactic acid which was used for checking the bacterial growth. A total number of 50 inocula were transferred in 10 Petri plates for each sample. Then the inoculated plates were incubated at room temperature $\left(25 \pm 2^{\circ} \mathrm{C}\right)$ for seven days. On the eighth day, the colonies of fungi were examined for mycelial growth, color and nature of the colony, sporulation, pigmentation as well as zonations.

Detailed morphological studies of the fungal isolates were made in order to determine their identification. The microscopic structural characters of the isolated fungi were recorded under Nikon D 5000 digital camera. Identification confirmed after examination of the morphology of conidia and conidiophores. Diagrammatic sketches of sporulating structures were also made by camera Lucida. Appropriate keys were consulted for identification of the fungi ${ }^{(17,18)}$. The isolates of $B$. sorokiniana were designated based on its location and source following Aminuzzaman et al.(19). For example, an isolate designated by JSVBjL-1, represents that this isolate was collected from district Joypurhat (J), Upazila Joypurhat Sadar (S), village Vutiapara (V) and from variety Bijoy (Bj), plant part used leaf (L), it was isolated, 1 denotes collection serial number. All specimens, included in the present study were preserved in Mycology and Plant Pathology Laboratory, Department of Botany, University of Dhaka, Bangladesh (Table 1).

A five mm mycelial disk was taken from the edge of the actively growing colonies and transferred to the Petri plates containing PDA to obtain pure culture of the pathogen. Mycelial compatibility grouping was performed as described by Schafer and Kohn ${ }^{(20)}$ and Minghe et al. ${ }^{(21)}$. Bipolaris sorokiniana isolates were paired on PDA medium maintain a distance of $3 \mathrm{~cm}$ between them and also from the edge of the Petri plate. A five $\mathrm{mm}$ mycelial disk of 7 days old PDA culture of each isolates from same and different cultural group was placed on the same Petri plate and incubate at room temperature $\left(25 \pm 2^{\circ} \mathrm{C}\right)$ for 21 - 28 days. After incubation the reaction between each isolate pair was evaluated. 
Table 1. Bipolaris sorokiniana isolates used in this experiment, their origin from different locations, source and cultural groups.

\begin{tabular}{|c|c|c|c|c|c|c|c|}
\hline $\begin{array}{l}\text { Sl. } \\
\text { No. }\end{array}$ & $\begin{array}{l}\text { Isolates } \\
\text { name }\end{array}$ & District & Upazilla & Village & Source & Variety & $\begin{array}{l}\text { Morphological } \\
\text { groups }\end{array}$ \\
\hline 1 & JSDSvL-01 & Joypurhat & Sadar & Doripara & Leaf & Saurav & Black-Mat \\
\hline 2 & JSDSvL-05 & $"$ & $"$ & $"$ & $"$ & $"$ & Greenish Ash-Fluffy \\
\hline 3 & JSDSvL-09 & $"$ & $"$ & $"$ & $"$ & $"$ & Pinkish White-Mat \\
\hline 4 & JSDSvL-18 & $"$ & $"$ & $"$ & $"$ & $"$ & Black-Mat \\
\hline 5 & JSDSvL-19 & $"$ & $"$ & $"$ & $"$ & $"$ & Whitish Ash-Mat \\
\hline 6 & JSDSvL-21 & $"$ & $"$ & $"$ & $"$ & $"$ & Blackish Ash-Mat \\
\hline 7 & JSDSvL-23 & $"$ & $"$ & $"$ & $"$ & $"$ & Whitish Ash-Mat \\
\hline 8 & JSDSvL-25 & $"$ & $"$ & $"$ & $"$ & $"$ & Ash-Mat \\
\hline 9 & JSDSvL-26 & $"$ & $"$ & $"$ & $"$ & $"$ & Whitish Ash-Mat \\
\hline 10 & JSDSvL-27 & $"$ & $"$ & $"$ & $"$ & $"$ & Whitish Ash-Mat \\
\hline 11 & JSDSvL-28 & $"$ & $"$ & $"$ & $"$ & $"$ & Ash-Mat \\
\hline 12 & JSDStL-01 & $"$ & $"$ & $"$ & $"$ & Shatabdi & Pinkish White-Mat \\
\hline 13 & JSDStL-06 & $"$ & $"$ & $"$ & $"$ & $"$ & Whitish Ash-Mat \\
\hline 14 & JSDStL-10 & $"$ & $"$ & $"$ & $"$ & $"$ & Black-Mat \\
\hline 15 & JSDPdL-02 & $"$ & $"$ & $"$ & $"$ & Prodip & Blackish Ash-Mat \\
\hline 16 & JSDBjL-03 & $"$ & $"$ & $"$ & $"$ & Bijoy & Brownish Ash-Fluffy \\
\hline 17 & JSVSvL-01 & $"$ & $"$ & Vutiapara & $"$ & Saurav & Brownish Ash-Fluffy \\
\hline 18 & JSVPdL-01 & $"$ & $"$ & $"$ & $"$ & Prodip & Black-Mat \\
\hline 19 & JSVPdL-04 & $"$ & $"$ & $"$ & $"$ & $"$ & Whitish Ash-Mat \\
\hline 20 & JSVPdL-05 & $"$ & $"$ & $"$ & $"$ & $"$ & Ash-Mat \\
\hline 21 & JSVPdL-07 & $"$ & $"$ & $"$ & $"$ & $"$ & Greenish Ash-Fluffy \\
\hline 22 & JSVPdL-08 & $"$ & $"$ & $"$ & $"$ & $"$ & Black-Fluffy \\
\hline 23 & JSVPdL-10 & $"$ & $"$ & $"$ & $"$ & Prodip & Blackish Ash-Mat \\
\hline 24 & JSVPdL-15 & $"$ & $"$ & $"$ & $"$ & $"$ & Blackish Ash-Mat \\
\hline 25 & JSVPdL-16 & $"$ & $"$ & $"$ & $"$ & $"$ & Brownish Ash-Fluffy \\
\hline 26 & JSVPdL-17 & $"$ & $"$ & $"$ & $"$ & $"$ & Blackish Ash-Mat \\
\hline 27 & JSVBjL-07 & $"$ & $"$ & $"$ & $"$ & Bijoy & Greenish Ash-Fluffy \\
\hline 28 & GJBKnL-01 & Gazipur & $"$ & BARI(Field) & $"$ & Kanchan & Black-Fluffy \\
\hline 29 & GJBKnL-02 & $"$ & $"$ & $"$ & $"$ & $"$ & Ash-Mat \\
\hline 30 & GJBKnL-03 & $"$ & $"$ & $"$ & $"$ & $"$ & Greenish-Ash-Fluffy \\
\hline 31 & GJBEnL-01 & $"$ & $"$ & $"$ & $"$ & En-113 & Black-Mat \\
\hline 32 & GJBEnL-02 & $"$ & $"$ & $"$ & $"$ & En-113 & Blackish Ash-Mat \\
\hline 33 & GJBKhL-01 & $"$ & $"$ & $"$ & $"$ & Khari & Ash-Mat \\
\hline 34 & GJBKIL-09 & $"$ & $"$ & $"$ & $"$ & Kalyansona & Whitish Ash-Mat \\
\hline 35 & GJBSrL-01 & $"$ & $"$ & $"$ & $"$ & Seri-82 & Brownish Ash-Fluffy \\
\hline 36 & PSVStL-02 & Pabna & Sathia & Vatikaya & $"$ & Shatabdi & Blackish Ash-Mat \\
\hline 37 & DiWRCL-10 & Dinajpur & Nashipur & WRC(Field) & $"$ & Ciano-T-79 & Blackish Ash-Mat \\
\hline 38 & DiWRBjL-01 & " & $"$ & $"$ & $"$ & Bijoy & Brownish Ash-Fluffy \\
\hline
\end{tabular}




\section{Results and Discussion}

Two hundred and ninety three isolates of Bipolaris sorokiniana collected during 20112014 from foliar and seed samples of wheat, among them 150 isolates were characterized on the basis of colony color and texture. The results exhibited considerable variation of the isolates. Results indicated that the colony color varied from black, ash, brown, green to white and mycelial growth type was effuse (fluffy) to suppress (mat) with regular to irregular growth patterns.

One hundred fifty isolates were classified in eight cultural types, such as - Black-Mat (B-M), Black-Fluffy (B-F), Ash-Mat (A-M), Brownish Ash-Fluffy (Br.A-F), Blackish AshMat (Bl.A-M), Whitish Ash-Mat (W.A-M), Greenish Ash-Fluffy (G.A-F) and Pinkish White-Mat (P.W-M) (Fig. 1). Among them twenty six isolates exhibited black colony color, where eighteen isolates were Black-Mat type and eight isolates were Black-Fluffy type, thirty seven Blackish Ash, ten Brownish Ash, twenty isolates were found in Ash and twenty one isolates in Whitish Ash group, thirty two isolates show Greenish Ash colony, while only four isolates showed albino colored colony or Pinkish White colony. Among them there cultural groups show fluffy growth - Black-Fluffy, Brownish Ash and Greenish Ash Fluffy. Total $66.67 \%$ of the isolates had suppress/mat type of colony while $33.34 \%$ showed fluffy type of growth on the medium. The maximum isolates exhibited Blackish Ash color followed by Greenish Ash, Black, Whitish Ash, Ash, and Pinkish White colored colony. Out of 150 isolates 37 isolates exhibited Blackish Ash-Mat (B.A-M), 32 Greenish Ash-Fluffy (G.A-F), 21 Whitish Ash-Mat (W.A-M), 20 Ash-Mat (A-M), 18 Black-Mat (B-M), 10 Brownish Ash-Fluffy (Br.A-F), 08 Black-Fluffy (B-F) and only 04 exhibited Pinkish White-Mat (P.W-M) colony. The percentage of each group (highest to lowest) are -24.67, 21.34, 14.0, 13.33, 12.0, 6.67, 5.33 and 2.67, respectively (Table 2).

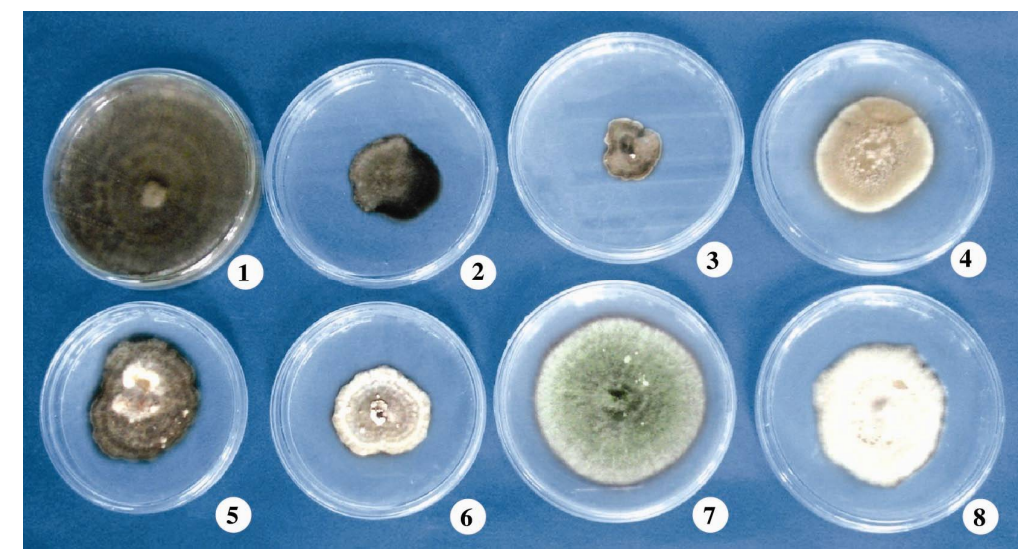

Fig. 1. Eight cultural groups of Bipolaris sorokiniana isolates based on colony colour and texture, 1. BlackMat (B-M) 2. Black- Fluffy (B-F) 3. Ash-Mat (A-M) 4. Brownish Ash-Fluffy (Br.A-F) 5. Blackish AshMat (Bl.A-M) 6. Whitish Ash Mat (WA-M), 7. Greenish Ash Fluffy (GA-F), 8. Pinkish White-Mat (PW-M). 
Table 2. Cultural variations of the selected isolates of Bipolaris sorokiniana.

\begin{tabular}{|c|c|c|c|c|c|}
\hline $\begin{array}{l}\text { Sl. } \\
\text { No. }\end{array}$ & $\begin{array}{l}\text { Cultural } \\
\text { group }\end{array}$ & $\begin{array}{l}\text { Detailed } \\
\text { description }\end{array}$ & $\begin{array}{l}\text { Code of B. sorokiniana } \\
\text { isolates }\end{array}$ & $\begin{array}{l}\text { No. of } \\
\text { isolates }\end{array}$ & $\%$ \\
\hline 1 & $\begin{array}{l}\text { Black- } \\
\text { Mat } \\
(\mathrm{B}-\mathrm{M})\end{array}$ & $\begin{array}{l}\text { Upper side Black, reverse side } \\
\text { black, Mat, no white dots, } \\
\text { Growth medium to fast, } \\
\text { Growth pattern regular. }\end{array}$ & $\begin{array}{l}\text { GJBKhL-08, GJBEnL-01, DiWRSIL-01, } \\
\text { KMKPdL-01, JSDStL-07, JSDStL-10, JSDSvL- } \\
\text { 01, JSDSvL-18, JSVBjL-08, JSVPdL-01, } \\
\text { GJB28S-04, GJB28S-05, GJB28S-06, GJB29S-02, } \\
\text { GJB29S-04, GJB29S-05, GJB29S-06, GJB29S-07 }\end{array}$ & 18 & 12.0 \\
\hline 2 & $\begin{array}{l}\text { Black- } \\
\text { Fluffy } \\
\text { (B-F) }\end{array}$ & $\begin{array}{l}\text { Upper side Black with ash } \\
\text { center and periphery is shiny } \\
\text { black, make it velvety, reverse } \\
\text { side black, Fluffy, no white } \\
\text { dots, Growth medium, } \\
\text { Growth pattern regular or } \\
\text { irregular. }\end{array}$ & $\begin{array}{l}\text { GJBKhL-16, GJBKnL-01, KVKBjL-01, PBKStL- } \\
\text { 01, JSDStL-11, JSDPdL-01, JSVStL-02, } \\
\text { JSVPdL-08 }\end{array}$ & 08 & 5.33 \\
\hline 3 & $\begin{array}{l}\text { Ash- } \\
\text { Mat } \\
(\text { A-M) }\end{array}$ & $\begin{array}{l}\text { Upper side Ash, reverse side } \\
\text { comparatively light ash, Mat, } \\
\text { many white sectors, Growth } \\
\text { medium, Growth pattern } \\
\text { irregular. }\end{array}$ & $\begin{array}{l}\text { GJBKhL-01, GJBK1L-01, GJBSnL-01, } \\
\text { DiWR25L-01, SSBStL-04, SSBStL-06, } \\
\text { DUC27L-02, DUC28L-02, CSFStL-01, JSDSvL- } \\
\text { 20, JSDSvL-22, JSDSvL-24, JSDSvL-25, } \\
\text { JSDSvL-28, JSVPdL-05, JSVPdL-06, GJBKnL- } \\
\text { 02, GJB26S-03, GJB28S-08, GJB28S-09 }\end{array}$ & 20 & 13.33 \\
\hline 4 & $\begin{array}{l}\text { Brownis } \\
\text { h Ash- } \\
\text { Fluffy } \\
\text { (Br.A-F) }\end{array}$ & $\begin{array}{l}\text { Upper side Brownish ash, } \\
\text { reverse side brownish ash } \\
\text { with black centre, Fluffy, no } \\
\text { white sectors, Growth slow to } \\
\text { medium, Growth pattern } \\
\text { regular. }\end{array}$ & $\begin{array}{l}\text { GJBSrL-01, DiWRStL-01, DiWRBjL-01, } \\
\text { DiWRBjL-03, JSDStL-02, JSDStL-03, JSDStL- } \\
\text { 05, JSDBjL-03, JSVPdL-16, JSVSvL-01 }\end{array}$ & 10 & 6.67 \\
\hline 5 & $\begin{array}{l}\text { Blackish } \\
\text { Ash-Mat } \\
\text { (Bl.A-M) }\end{array}$ & $\begin{array}{l}\text { Upper side Blackish ash, } \\
\text { reverse side blackish ash, Mat, } \\
\text { white sectors present in few } \\
\text { isolates, Growth slow to } \\
\text { medium, Growth pattern } \\
\text { regular or irregular. }\end{array}$ & $\begin{array}{l}\text { DiWRBIL-01, DiWRPdL-02, DiWR26L-02, } \\
\text { DiWRCL-10, GJBEnL-02, PSVStL-02, JSDPdL- } \\
\text { 02, JSDPdL-03, JSDPdL-04, JSDPdL-06, } \\
\text { JSDPdL-12, JSDPdL-13, JSDPdL-14, JSDPdL- } \\
\text { 16, JSDSvL-02, JSDSvL-03, JSDSvL-21, } \\
\text { JSVBjL-01, JSVBjL-02, JSVBjL-09, JSVBjL-10, } \\
\text { JSVPdL-02, JSVPdL-03, JSVPdL-09, JSVPdL- } \\
\text { 10, JSVPdL-11, JSVPdL-12, JSVPdL-13, } \\
\text { JSVPdL-14, JSVPdL-15, JSVPdL-17, GJB25S- } \\
\text { 01, GJB26S-02, GJB28S-01, GJB29S-01, } \\
\text { GJB29S-03, GJB30S-01 }\end{array}$ & 37 & 24.67 \\
\hline 6 & $\begin{array}{l}\text { Whitish } \\
\text { Ash-Mat } \\
\text { (W.A-M) }\end{array}$ & $\begin{array}{l}\text { Upper side Whitish ash, } \\
\text { reverse side ash, Mat/Fluffy, } \\
\text { many white sectors, Growth } \\
\text { medium, Growth pattern } \\
\text { irregular. }\end{array}$ & $\begin{array}{l}\text { GJBK1L-09, GJBK1L-12, GJBEnL-03, SSBStL- } \\
\text { 03, SSBStL-05, SSVSvL-01, SSVSvL-06, } \\
\text { SSVSvL-08, SSVPdL-01, KVFStL-01, JSDStL- } \\
\text { 06, JSDBjL-02, JSDSvL-04, JSDSvL-19, } \\
\text { JSDSvL-23, JSDSvL-26, JSDSvL-27, JSVPdL- } \\
\text { 04, GJB25S-02, GJB28S-02, GJB28S-03 }\end{array}$ & 21 & 14.0 \\
\hline
\end{tabular}




\begin{tabular}{|c|c|c|c|c|c|}
\hline 7 & $\begin{array}{l}\text { Greenis } \\
\text { h Ash- } \\
\text { Fluffy } \\
\text { (G.A-F) }\end{array}$ & $\begin{array}{l}\text { Upper side Greenish ash, } \\
\text { center to periphery colour is } \\
\text { dark to light, reverse side ash } \\
\text { with black center and ray } \\
\text { pattern, mainly Fluffy, no } \\
\text { white sectors, Growth } \\
\text { medium to fast, Growth } \\
\text { pattern regular. }\end{array}$ & $\begin{array}{l}\text { GJBKhL-18, DiWRAnL-01, DiWRKnL-01, } \\
\text { DiWRAgL-01, GJBEnL-04, SSBStL-01, } \\
\text { SSBStL-02, SSBStL-07, SSVSvL-02, SSVSvL- } \\
\text { 03, SSVSvL-04, SSVSvL-05, SSVSvL-07, } \\
\text { SSVPdL-02, SSBBjL-01, SSBBjL-02, SSBBjL- } \\
\text { 03, SSBBjL-04, SSBBjL-05, DUC25L-01, } \\
\text { DUC25L-02, DUC25L-03, DUC25L-04, } \\
\text { DUC26L-01, DUC26L-05, PBKBjL-02, JSDStL- } \\
\text { 04, JSDSvL-05, JSVBjL-07, JSVPdL- } \\
\text { 07,GJBKnL-03, GJB30S-02 }\end{array}$ & 32 & 21.34 \\
\hline 8 & $\begin{array}{l}\text { Pinkish } \\
\text { White- } \\
\text { Mat } \\
\text { (P.W-M) }\end{array}$ & $\begin{array}{l}\text { Upper side Pinkish white, } \\
\text { reverse side same, Mat, no } \\
\text { white sectors, Growth } \\
\text { medium to fast, Growth } \\
\text { pattern regular to irregular. }\end{array}$ & CSFBjL-01, PBCBjL-03, JSDStL-01, JSDSvL-09 & 04 & 2.67 \\
\hline & & & Total & 150 & 100 \\
\hline
\end{tabular}

Variation was also noticed in colony margin and colony growth patterns. Both regular and irregular type's colony growth patterns were observed in the isolates. Most of the fluffy type colonies showed regular growth and most of the mat type colonies showed irregular growth. Though some exceptions were also present. Two types of colony margin were recorded for the isolates. These were - smooth and wavy colony margin. Most of the fluffy type colonies showed smooth colony margin, where most of the mat type colonies showed wavy colony margin, there were also some exceptions. Wavy colony margins were remarkable in Blackish Ash-Mat (B.A-M) type, but Black-Mat (B-M) type colony showed smooth colony margins. Another remarkable character was presence of white sectors on the colony. Black colored colony that means Black-Mat (B$\mathrm{M})$ and Black-Fluffy (B-F) type's isolates showed no white sectors. Fluffy typed colonies that mean Brownish Ash-Fluffy (Br.A-F) and Greenish Ash-Fluffy (G.A-F) and also Pinkish White-Mat (P.W-M) isolates exhibited no white sectors. Mainly three types of isolates exhibited white sectors on the dorsal surface of the colonies. Those were Ash-Mat (A-M), Blackish Ash-Mat (Bl.A-M) and Whitish Ash-Mat (W.A-M). But number of sectors, their shape and size were different from one another. Sometimes number of sectors was easily countable but others that was not possible. In some cases white sectors were very tiny in size, shape may be circular or ellipsoidal, very easy for countdown, but in other cases sectors size were large, later mixed with each other, then countdown was not possible. So, these characters were recorded for individual isolates as morphological variability, along with other characteristics, such as conidial length-breadth, conidial abundance, size of conidiophores etc. After inoculation and growth of two different isolates on one plate, it was observed that as the colony size increased, their mycelia came in contact with each other. Total thirty one confrontations were made. Three different behaviors in hyphal overlapping region were observed, which allowed us to 
classify the confrontations as compatible, partially compatible and incompatible (Figs 2, $3,4,5$ and 6 ). Of 31 confrontations, 8 were compatible, 6 were partially compatible and 17 were incompatible (Table 3).
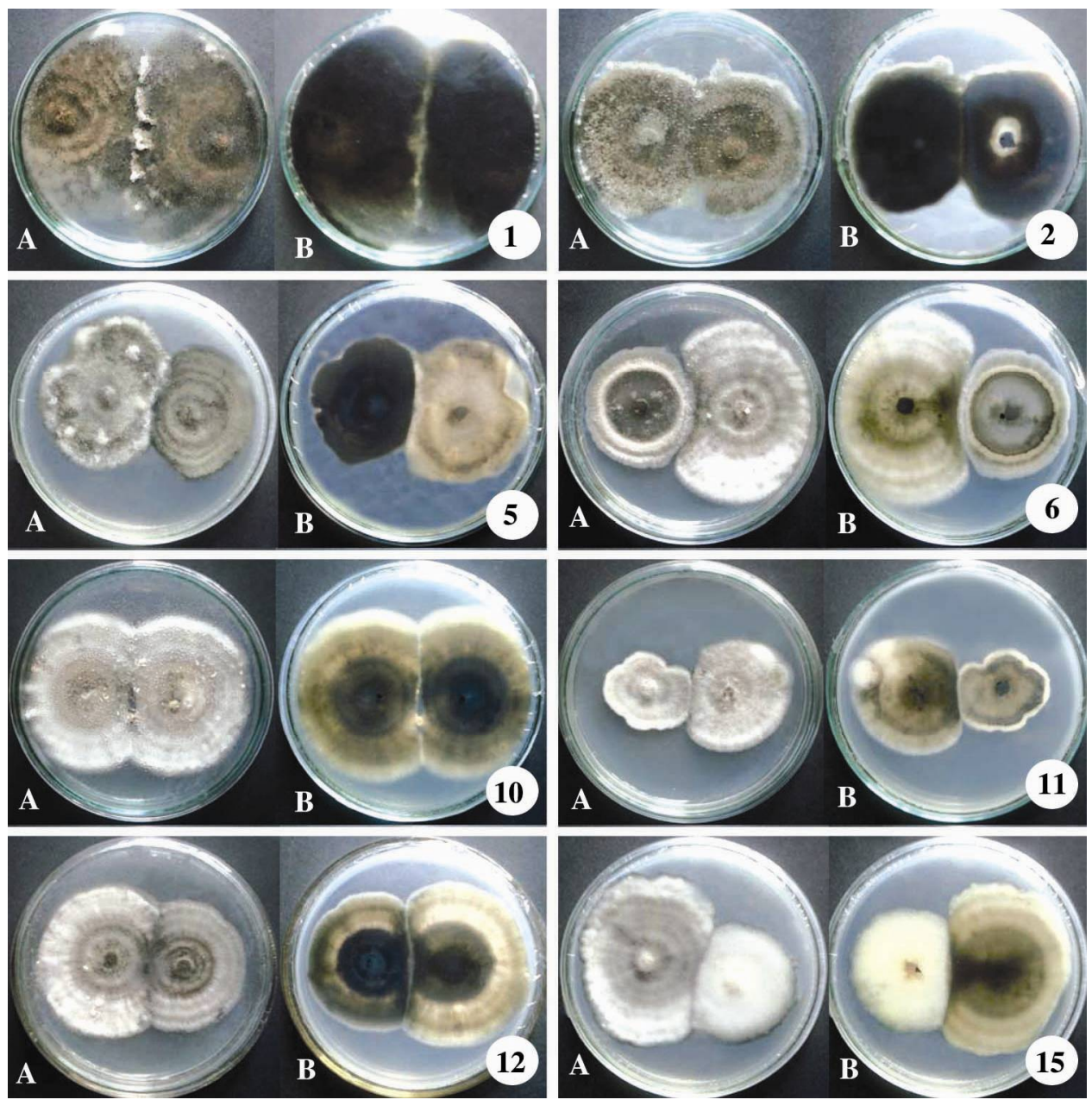

Fig. 2. Compatible confrontation of eight pairs of Bipolaris sorokiniana isolates 1A-B, 2A-B, 5A-B, 6A-B, 10A-B, 11A-B, 12A-B and 15A-B (A shows upper- and B lower view).

In the compatible confrontations, it was possible to observe the overlapping of hyphae in even part of the colonies. In the partially compatible confrontations, initially, intermingling of the mycelia of two incompatible isolates took place but later on lysis of mycelia of two isolates and development of a clear zone was observed at the region of interaction (mycelial contact). The mycelial incompatibility reaction is shown in Fig. 4 and 5 (top and bottom). The results from the confrontations showed that $55.0 \%$ of them were vegetatively incompatible. Therefore, the study revealed that mycelial compatibility 
reaction could be used to distinguish morphologically different isolates belonging to the same species. Slippers et al.(22) analyzing incompatibility, noted that antagonistic reactions are more common between related organisms than between genetically or geographically distant isolates.
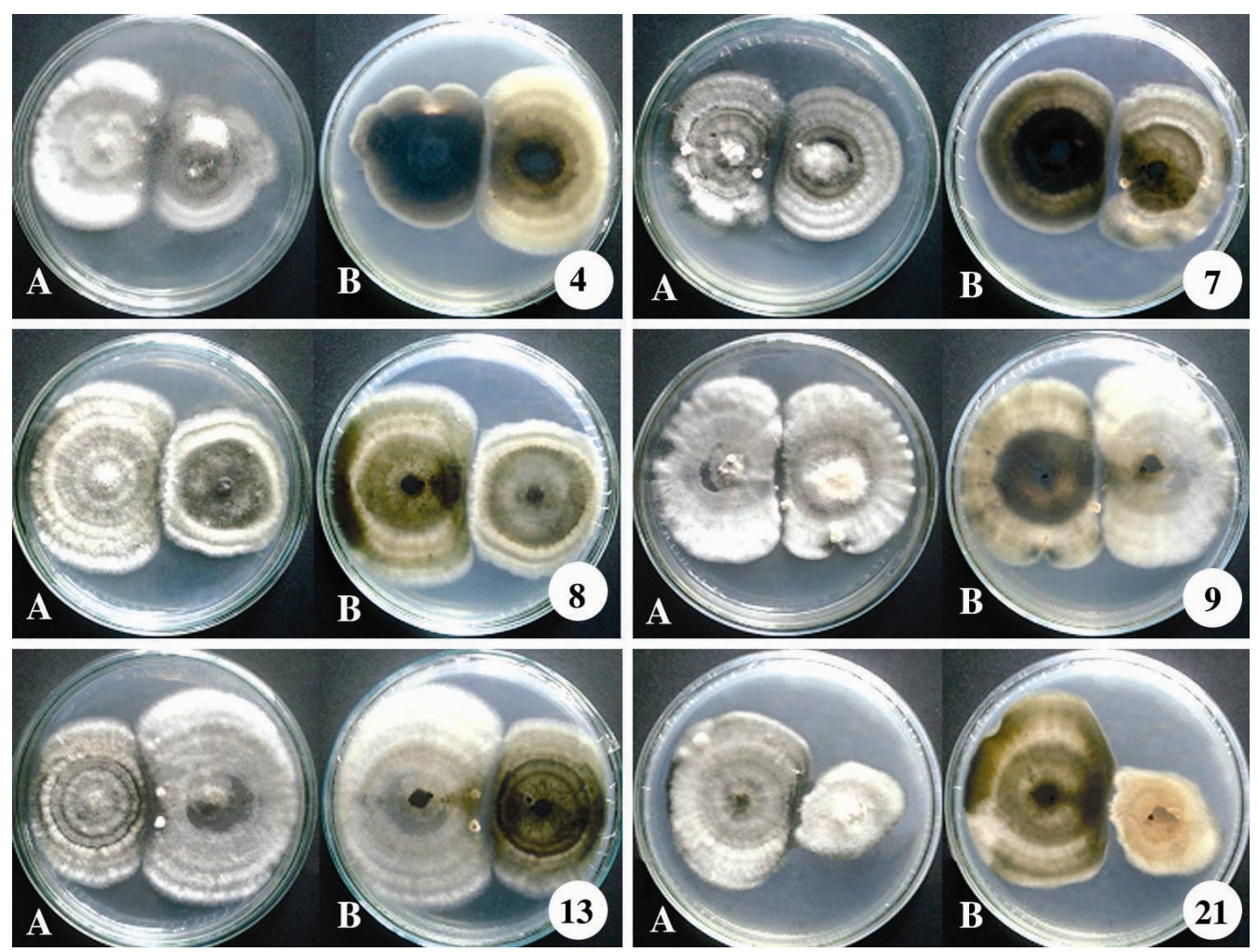

Fig. 3. Partially compatible confrontation of six pairs of Bipolaris sorokiniana isolates 4A-B, 7A-B, 8A-B, 9A-B, 13A-B and 21A-B (A shows upper- and B lower view).

Considerable numbers of research work have been conducted all over the world on the variation of the pathogen. Ahmed et al.(23) obtained physiological and morphological variation among 27 isolates of B. sorokiniana collected from 14 districts in Bangladesh. Colonies were ash brown, olive green, light green or dark green in color with regular or wavy margins, fluffy, spread or velvety texture and with or without sector. Aminuzzaman et al.(19) differentiated 86 isolates into nine cultural groups based on their colony morphology and colony color. Maximum number of 34 isolates produced effuse black regular colony with a frequency of $39.53 \%$, where 29 isolates produced effuse black irregular colony with a frequency of $33.72 \%$ of collected isolates. Ahmed collected 262 isolates of B. sorokiniana from 16 major wheat growing areas of Bangladesh and he grouped these isolates in 13 physiological groups based on their cultural characteristics ${ }^{(24)}$. 

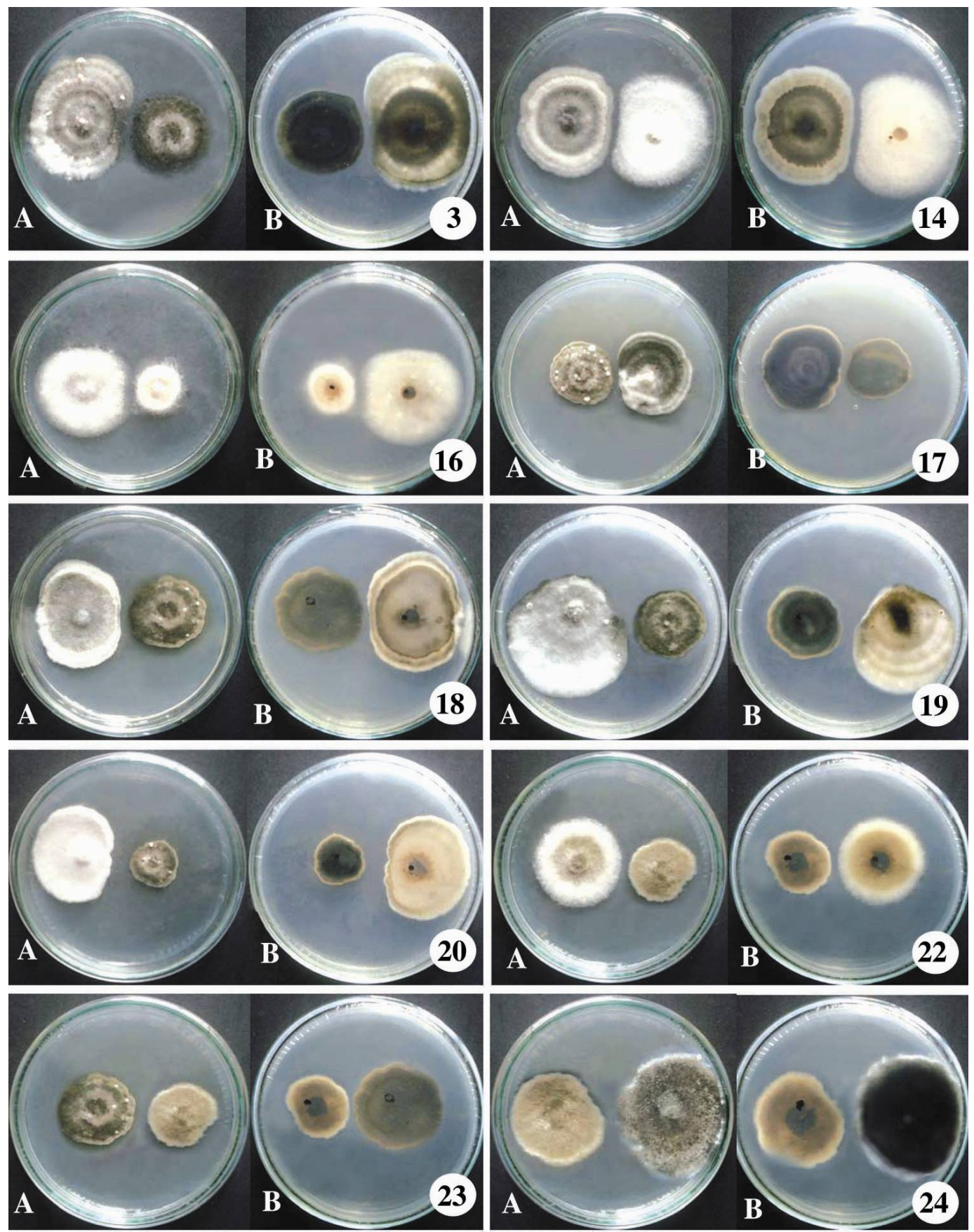

Fig. 4. Incompatible confrontation of ten pairs of Bipolaris sorokiniana isolates 3A-B, 14A-B, 16A-B, 17A-B, 18A-B, 19A-B, 20A-B, 22A-B, 23A-B and 24A-B (A shows upper- and B lower view). 

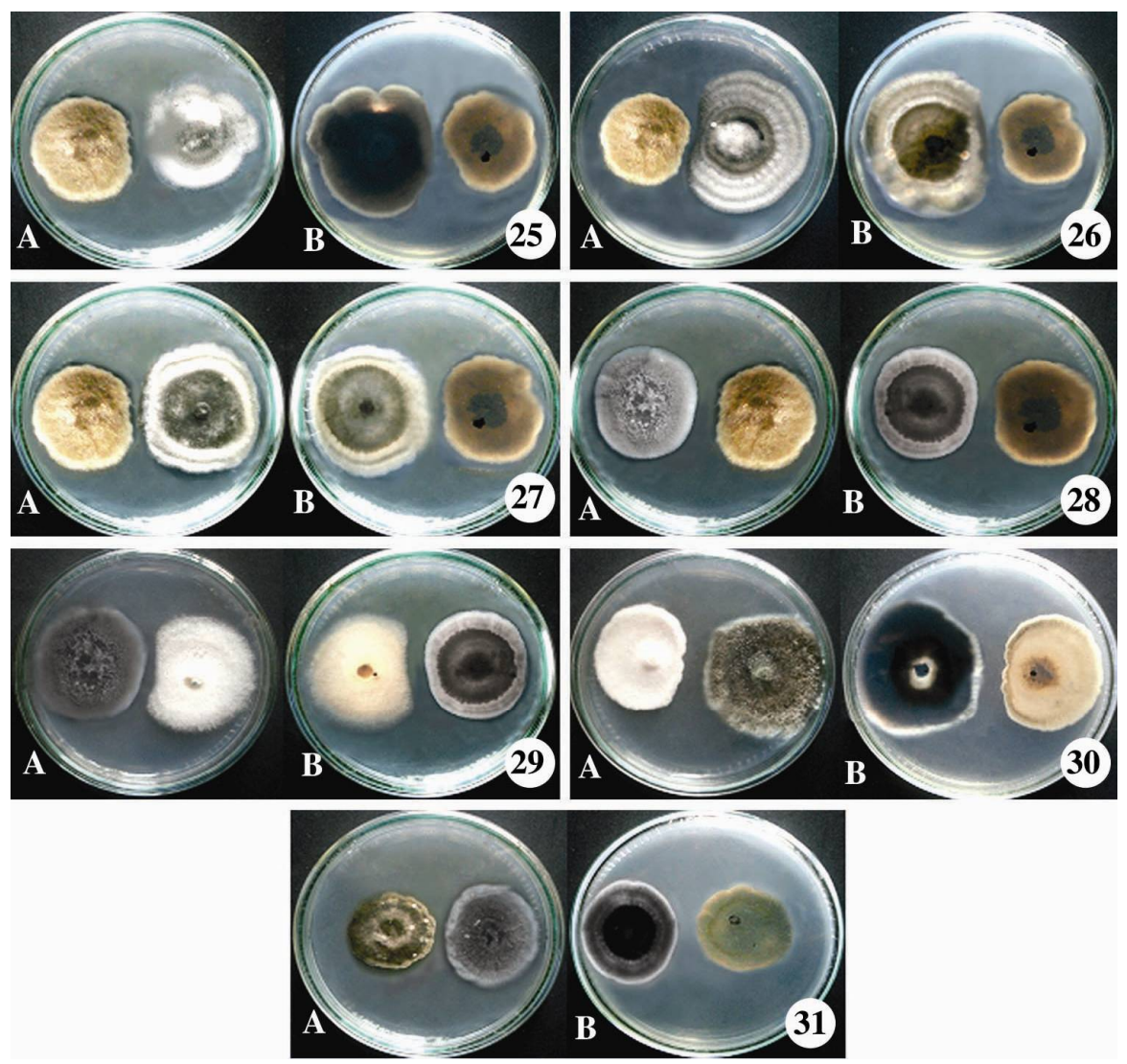

Fig. 5. Incompatible confrontation of seven pairs of Bipolaris sorokiniana isolates 25A-B, 26A-B, 27A-B, 28A-B, 29A-B, 30A-B and 31A-B (A shows upper- and B lower view).

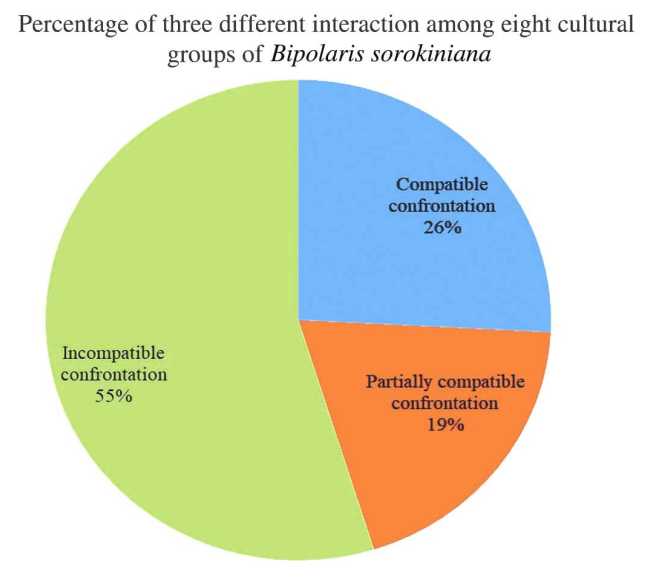

Fig. 6. Pie chart shows the frequency percentage of three different confrontations as compatible, partially compatible and incompatible. 
Table 3. Compatibility between different cultural groups.

\begin{tabular}{|c|c|c|c|c|}
\hline $\begin{array}{l}\text { S1 } \\
\text { no. }\end{array}$ & $\begin{array}{l}\text { Isolate name } \\
\text { pairs }\end{array}$ & $\begin{array}{l}\text { Cultural group } \\
\text { pairs }\end{array}$ & $\begin{array}{l}\text { District } \\
\text { pairs }\end{array}$ & $\begin{array}{l}\text { Compatible/ } \\
\text { incompatible }\end{array}$ \\
\hline 1 & $\begin{array}{l}\text { JSDSvL-01 - } \\
\text { JSDSvL-18 }\end{array}$ & Black-Mat - Black-Mat & Joypurhat-Joypurhat & Compatible \\
\hline 2 & $\begin{array}{l}\text { JSDSvL-01 - } \\
\text { JSDStL-10 }\end{array}$ & Black-Mat - Black-Mat & $"$ & $"$ \\
\hline 3 & $\begin{array}{l}\text { JSVPdL-10 - } \\
\text { GJBKnL-01 }\end{array}$ & Blackish Ash-Mat - Black-Fluffy & Joypurhat- Gazipur & Incompatible \\
\hline 4 & $\begin{array}{l}\text { JSVPdL-05 - } \\
\text { JSVPdL-08 }\end{array}$ & Ash-Mat - Black-Fluffy & Joypurhat-Joypurhat & $\begin{array}{l}\text { Partially } \\
\text { compatible }\end{array}$ \\
\hline 5 & $\begin{array}{l}\text { JSVPdL-01 - } \\
\text { JSDSvL-21 }\end{array}$ & Black-Mat - Blackish Ash-Mat & $"$ & Compatible \\
\hline 6 & $\begin{array}{l}\text { GJBKhL-01 - } \\
\text { GJBEnL-02 }\end{array}$ & Ash-Mat - Blackish Ash-Mat & Gazipur-Gazipur & Compatible \\
\hline 7 & $\begin{array}{l}\text { JSVPdL-15 - } \\
\text { JSDSvL-21 }\end{array}$ & $\begin{array}{l}\text { Blackish Ash-Mat - Blackish Ash- } \\
\text { Mat }\end{array}$ & Joypurhat-Joypurhat & $\begin{array}{l}\text { Partially } \\
\text { compatible }\end{array}$ \\
\hline 8 & $\begin{array}{l}\text { GJBKnL-02 - } \\
\text { PSVStL-02 }\end{array}$ & Ash-Mat - Blackish Ash-Mat & Gazipur-Pabna & $"$ \\
\hline 9 & $\begin{array}{l}\text { JSDSvL-28 - } \\
\text { JSDSvL-25 }\end{array}$ & Ash-Mat - Ash-Mat & Joypurhat-Joypurhat & " \\
\hline 10 & $\begin{array}{l}\text { JSVPdL-04 - } \\
\text { JSDSvL-27 }\end{array}$ & Whitish Ash-Mat - Whitish Ash-Mat & $"$ & Compatible \\
\hline 11 & $\begin{array}{l}\text { JSDSvL-19- } \\
\text { JSDSvL-23 }\end{array}$ & Whitish Ash-Mat - Whitish Ash-Mat & $"$ & $"$ \\
\hline 12 & $\begin{array}{l}\text { JSDSvL-25 - } \\
\text { JSDSvL-26 }\end{array}$ & Ash-Mat - Whitish Ash-Mat & $"$ & $"$ \\
\hline 13 & $\begin{array}{l}\text { JSDSvL-26 - } \\
\text { JSVPdL-17 }\end{array}$ & Whitish Ash-Mat - Blackish Ash-Mat & $"$ & $\begin{array}{l}\text { Partially } \\
\text { compatible }\end{array}$ \\
\hline 14 & $\begin{array}{l}\text { JSDStL-01 - } \\
\text { DiWRCL-10 }\end{array}$ & $\begin{array}{l}\text { Pinkish White-Mat - Blackish Ash- } \\
\text { Mat }\end{array}$ & Joypurhat-Dinajpur & Incompatible \\
\hline 15 & $\begin{array}{l}\text { JSDSvL-09- } \\
\text { JSDStL-06 }\end{array}$ & $\begin{array}{l}\text { Pinkish White-Mat - Whitish Ash- } \\
\text { Mat }\end{array}$ & Joypurhat- Joypurhat & Compatible \\
\hline 16 & $\begin{array}{l}\text { JSDStL-01 - } \\
\text { JSDSvL-09 }\end{array}$ & $\begin{array}{l}\text { Pinkish White-Mat - Pinkish White- } \\
\text { Mat }\end{array}$ & $"$ & Incompatible \\
\hline 17 & $\begin{array}{l}\text { JSDPdL-02 - } \\
\text { JSDSvL-05 }\end{array}$ & $\begin{array}{l}\text { Blackish Ash-Mat - Greenish Ash- } \\
\text { Fluffy }\end{array}$ & $"$ & $"$ \\
\hline 18 & $\begin{array}{l}\text { GJBKhL-01 - } \\
\text { JSVPdL-07 }\end{array}$ & Ash-Mat - Greenish Ash-Fluffy & $"$ & $"$ \\
\hline 19 & $\begin{array}{l}\text { GJBK1L-09- } \\
\text { JSVBjL-07 }\end{array}$ & $\begin{array}{l}\text { Whitish Ash-Mat - Greenish Ash- } \\
\text { Fluffy }\end{array}$ & " & $"$ \\
\hline 20 & $\begin{array}{l}\text { JSDStL-01 - } \\
\text { GJBKnL-03 }\end{array}$ & $\begin{array}{l}\text { Pinkish White-Mat - Greenish Ash- } \\
\text { Fluffy }\end{array}$ & Joypurhat-Gazipur & $"$ \\
\hline 21 & $\begin{array}{l}\text { JSDSvL-09- } \\
\text { JSDSvL-28 }\end{array}$ & Pinkish White-Mat - Ash-Mat & Joypurhat-Joypurhat & $\begin{array}{l}\text { Partially } \\
\text { compatible }\end{array}$ \\
\hline
\end{tabular}

(Contd.) 


\begin{tabular}{|c|c|c|c|c|}
\hline 22 & $\begin{array}{l}\text { JSDBjL-03 - } \\
\text { JSVBjL-07 }\end{array}$ & $\begin{array}{l}\text { Brownish Ash-Fluffy - Greenish- } \\
\text { Ash-Fluffy }\end{array}$ & Joypurhat-Joypurhat & Incompatible \\
\hline 23 & $\begin{array}{l}\text { GJBKnL-03 - } \\
\text { DiWRBjL-01 }\end{array}$ & $\begin{array}{l}\text { Greenish-Ash-Fluffy - Brownish } \\
\text { Ash-Fluffy }\end{array}$ & Joypurhat-Dinajpur & " \\
\hline 24 & $\begin{array}{l}\text { JSVSvL-01 - } \\
\text { JSDSvL-18 }\end{array}$ & Brownish Ash-Fluffy - Black-Mat & Joypurhat-Joypurhat & $"$ \\
\hline 25 & $\begin{array}{l}\text { JSVPdL-16 - } \\
\text { JSVPdL-08 }\end{array}$ & Brownish Ash-Fluffy- Black-Fluffy & $"$ & $"$ \\
\hline 26 & $\begin{array}{l}\text { DiWRBjL-01 - } \\
\text { DiWRCL-10 }\end{array}$ & $\begin{array}{l}\text { Brownish Ash-Fluffy - Blackish Ash- } \\
\text { Mat }\end{array}$ & Dinajpur - Dinajpur & $"$ \\
\hline 27 & $\begin{array}{l}\text { JSVPdL-16 - } \\
\text { JSDSvL-28 }\end{array}$ & Brownish Ash-Fluffy - Ash-Mat & Joypurhat-Joypurhat & $"$ \\
\hline 28 & $\begin{array}{l}\text { GJBKnL-02 - } \\
\text { GJBSrL-01 }\end{array}$ & Ash-Mat - Brownish Ash-Fluffy & Gazipur - Gazipur & $"$ \\
\hline 29 & $\begin{array}{l}\text { GJBKnL-02 - } \\
\text { JSDStL-01 }\end{array}$ & Ash-Mat - Pinkish White-Mat & Gazipur - Joypurhat & $"$ \\
\hline 30 & $\begin{array}{l}\text { JSDSvL-09 - } \\
\text { GJBEnL-01 }\end{array}$ & Pinkish White-Mat - Black-Mat & Joypurhat-Gazipur & $"$ \\
\hline 31 & $\begin{array}{l}\text { JSDSvL-05 - } \\
\text { JSDSvL-28 }\end{array}$ & Greenish-Ash-Fluffy - Ash-Mat & Joypurhat-Joypurhat & $"$ \\
\hline
\end{tabular}

In the results higher frequency phenotypic variations indicating random distribution of $B$. sorokiniana throughout the country. It is highly likely that sexual reproduction take place in B. sorokiniana, although there is only one evidence of sexual reproduction in nature from this fungus ${ }^{(25)}$. Tinline et al. ${ }^{(26)}$ showed that perithecia were formed when monoconidial isolates of $B$. sorokiniana were paired in culture. Later Tinline ${ }^{(27)}$ established that two compatibility groups (a \& A) of the fungus occurred in Canada and that perithecia were produced only between pairs comprising a member from each group. Jones ${ }^{(28)}$ also found same results and two compatibility groups (a and A) of B. sorokiniana among Australian isolates. Poloni et al.(29) studied 35 isolates of B. sorokiniana from different regions of Brazil and other countries and categorized into five morphological groups having black fluffy growth with white sectors, black fluffy growth, gray cottony growth, white cottony growth and white suppressed growth. They also studied compatibility/ncompatibility reaction among them. Eighteen of 31 confronta-tions showed vegetative incompatibility. The results obtained with different culture media for the vegetative compatibility/incompatibility genotypes suggested that the type of substratum influences these reactions. The specific environmental and nutritional requirements for the production of perithecia in culture are established(27,30,31). However high level of variability in phenotypes among isolates indicated that sexual reproduction might have occurred very infrequently in nature. If it did occur, it could result in the production of new strains of the fungus which may be of even greater economic importance to the researchers than the existing strains. However, the extreme diversity of 
cultural variation suggested that the variation might arise due to mutations, heterokaryosis, parasexuality and other recombination mechanisms.

\section{Acknowledgement}

The first author (SM) gratefully acknowledges the financial support by the Ministry of Science and Technology, Government of the People's Republic of Bangladesh through NST fellowship.

\section{References}

1. BBS 2017. Statistical Year Book of Bangladesh. Bangladesh Bureau of Statistics. Ministry of Planning, Government of Bangladesh. pp. 78-79.

2. Talukdar MJ 1974. Plant diseases in Bangladesh. Bangladesh J. Agril. Res. 1(1): 61-86.

3. Ahmed HU 1986. Prevailing wheat diseases in Bangladesh. In: Anonymous (ed.). Third National Wheat Training Workshop, Wheat Research Centre, BARI, Joydebpur, Gazipur. pp. 124-134.

4. Sadat MA and J Choi 2016. Wheat blast: A new fungal inhabitant to Bangladesh threatening world wheat production. Bangladesh J. Pl. Pathol. 33(2): 103-108.

5. Ahmed SM and CA Meisner 1996. Wheat research and development of Bangladesh, CIMMYT Bangladesh. pp. 85.

6. Zillinsky FJ 1983. Common Diseases of Small Grain Cereals: A Guide to Identification. CIMMYT, Mexico DF, Mexico. CIMMYT. pp. 141.

7. Wiese MV 1998. Compendium of Wheat Diseases. (3rd Ed). APS Press, St. Paul Minnesota, USA. pp. 112.

8. Saari EE 1998. Leaf blight diseases and associated soil borne fungal pathogens of wheat in south and Southeast Asia. In: Helminthosporium Blight of Wheat: Spot Blotch and Tan Spot. E Duveiller, HJ Dubin, J Reeves and A McNab (Eds). Mexico DF, Mexico. CIMMYT. pp. 37-51.

9. Alam KB, PK Malaker, MA Shaheed, MU Ahmed, F Ahmed and MS Haque 1995. Yield loss assessment of wheat due to Bipolaris leaf blight in Bangladesh. Bangladesh J. Pl. Pathol. 11(1\&2): 35-38.

10. Singh RV, AK Singh and SP Singh 1997. Distribution of pathogens causing foliar blight of wheat in India and neighbouring countries. In: Helminthosporium Blight of Wheat: Spot blotch and Tan Spot (Eds. Duveiller E, Dubin HJ, Reeves J and McNab A). 9-14 February, CIMMYT, Mexico. pp. 59-62.

11. Kaufmann PJ and GJ Weidemann 1996. Plant Dis. 80: 1289-1293.

12. Tinline RD 1962. Cochliobolus sativus. V. Heterocariosis and parasexuality. Can. J. Bot. 40: 425437.

13. Saupe SJ 2000. Molecular genetics of heterokaryon incompatibility in filamentous ascomycetes. Microbial Mol. Boil. Rev. 64: 489-502.

14. Glass NL, DJ Jacobson and PKT Shiu 2000. The genetics of hyphal fusion and vegetative incompatibility in filamentous ascomycete fungi. Annu. Rev. Genet. 34: 165-186. 
15. Poloni A, MV Muller, I Pessi and ST Van Der Sand 2008. Analysis of morphological and growth rate variability of polyconidial and monoconidial cultures of Bipolaris sorokiniana. Current Microbiology 16(1): 52-63.

16. CAB (Commonwealth Agricultural Bureau) 1968. Plant Pathologist's Pocket Book. $1^{\text {st }}$ edition. The Commonwealth Mycological Institute, England. pp. 267.

17. Ellis MB 1971. Dematiaceous Hyphomycetes. Commonwealth Mycological Institute, England. pp 608.

18. Chidambaram P, SB Mathur and P Neergaard 1973. Identification of seed-borne Drechslera species. Friesia 10(3): 165-207.

19. Aminuzzaman FM, I Hossain and F Ahmed 2010. Cultural Variation and Pathogenicity of Bipolaris Sorokiniana on wheat in Bangladesh. Int. J. Agric. Enviornment and biotechnology 3(1): 207-216.

20. Schafer MR and LM Kohn 2006. An optimized method for mycelial compatibility testing in Sclerotinia sclerotiorum. Mycologia 98(4): 593-597.

21. Minghe Mo, Li Ziqin and WG ChenRu Li Yaand Dilantha Ferando 2010. Analysia of mycelial compatibility and pathogenicity of Sclerotium isolates from oilseed crops in Canada, China, USA and England. Plant Protection: Disease. 65: 190-193.

22. Slippers B, MJ Wingfield, BD Wingfield and TA Coutinho 2001. Population structure and possible origin of Amylostereum areolatum in South Africa. Plant Pathol. 50: 206-210.

23. Ahmed AU, MZ Rahman, KA Bhuiyan and IH Mian 1997. Variation in isolates of Bipolaris sorokiniana from wheat. Bangladesh J. Pl. Pathol. 13(1\&2): 29-36.

24. Ahmed F 2001. Studies on pathogenicity of Bipolaris sorokiniana and its effect on grain filling of wheat in Bangladesh. Ph.D. Thesis. Department of Plant Pathology, Bangladesh Agriculture University, Mymensingh.

25. Raemaekers RH 1985. In: Wheats for more tropical environments. A proceedings of a symposium. Mexico DF, Mexico. CIMMYT. pp. 201-203.

26. Tinline RD, BJ Sallans and PM Simmonds 1950. The perithecial stage of Helminthosporium sativum. Proc. Can. Phytopath. Soc. 17: 16 [Abstr.]

27. Tinline RD 1951. Studies on the perfect stage of Helminthosporium sativum. Can. J. Bot. 29: 467478.

28. Jones LC 1971. Studies of compatibility in Australian isolates of Bipolaris sorokiniana. Aust. J. boil.Sci.

29. Poloni A, IS Pessi, APG Frazzon and ST Van Der Sand 2009b. Vegetative incompatibility among monoconidial isolates of Bipolaris sorokiniana. Current Microbiology 58(2): 153-158.

30. Shoemaker RA 1955. Biology, cytology and taxonomy of Cochliobolus sativus. Can. J. Bot. 33: 562-576.

31. Tinline RD and Dickson JG 1958. Cochliobolus sativus I Perithecial development and the inheritance of spore color and mating type. Mycologia 50: 697-706. 